\title{
1 A chemotactic sensor controls Salmonella-host cell interaction
}

2 Stefanie Hoffmann ${ }^{1}$, Kathrin Gendera ${ }^{1}$, Christiane Schmidt ${ }^{1}$, Peter Kirchweger ${ }^{2 \#}$, Axel Imhof ${ }^{3}$, Christian

3 Bogdan $^{4}$, Yves A. Muller ${ }^{2}$, Michael Hensel $^{5}$, Roman G. Gerlach ${ }^{1,4^{*}}$

4

$5 \quad{ }^{1}$ Project Group 5, Robert Koch Institute, Wernigerode, Germany

62 Division of Biotechnology, Department of Biology, Friedrich-Alexander-Universität Erlangen-Nürnberg

7 (FAU), Erlangen, Germany

$8{ }^{3}$ BioMedical Center and Center for Integrated Protein Sciences Munich, Ludwig-Maximilians-Universität

9 München, München, Germany

$10{ }^{4}$ Mikrobiologisches Institut - Klinische Mikrobiologie, Immunologie und Hygiene, Universitätsklinikum 11 Erlangen and Friedrich-Alexander-Universität Erlangen-Nürnberg, Erlangen, Germany

$12{ }^{5}$ Abteilung Mikrobiologie, Universität Osnabrück, Osnabrück, Germany and CellNanOs - Center for 13 Cellular Nanoanalytics Osnabrück, Universität Osnabrück, Osnabrück, Germany \#present address: Department of Chemical and Structural Biology, Faculty of Chemistry, Weizmann Institute of Science, Rehovot, Israel

${ }^{*}$ Corresponding author: Roman G. Gerlach

Email: roman.gerlach@uk-erlangen.de

Author Contributions: R.G.G., S.H., M.H. and Y.A.M. designed research; S.H., K.G., C.S. and P.K. performed research; R.G.G., S.H. and A.I. analyzed the data; and R.G.G., C.B., M.H. and Y.A.M. wrote the paper.

Keywords: Salmonella, chemotaxis, adhesion, aspartate 


\section{Abstract}

Intimate cell contact and subsequent type three secretion system-dependent cell invasion are key steps in host colonization of Salmonella. Adhesion to complex glycostructures at the apical membrane of polarized cells is mediated by the giant adhesin SiiE. This protein is secreted by a type 1 secretion system (T1SS) and needs to be retained at the bacterial surface to exert its adhesive function. Here, we show that SiiE surface expression was linked to the presence of L-aspartate sensed by the Salmonellaspecific methyl-accepting chemotaxis protein CheM. Bacteria lacking CheM were attenuated for invasion of polarized cells, whereas increased invasion was seen with Salmonella exposed to the nonmetabolizable aspartate analog $\alpha-$ methyl-D, L-aspartate (MeAsp). While components of the chemotaxis phosphorelay or functional flagella were dispensable for the increased invasion, CheM directly interacted with proteins associated with the SiiE T1SS arguing for a novel non-canonical signaling mechanism. As a result, CheM attractant signaling caused a shift from secreted to surface-retained and adhesion-competent SiiE. Thus, CheM controls the virulence function of SiiE in a precise spatiotemporal fashion depending on the host micro-milieu. 


\section{INTRODUCTION}

Many pathogenic bacteria strongly rely on their ability to get into close contact to eukaryotic host cell surfaces. By means of different adhesins, they are able to colonize mucosal surfaces or invade cells and establish their niches in host organisms. Salmonella enterica subsp. enterica serovar Typhimurium (STM) is a pathogen that is capable to infect diverse hosts and usually causes a self-limiting gastrointestinal infection. STM can invade non-phagocytic cells by deploying a type III secretion system (T3SS) that is encoded by the Salmonella pathogenicity island 1 (SPI-1) (1). An intimate contact between the pathogen and the host cell is essential for the subsequent translocation of effector molecules by the SPI-1-dependent T3SS (T3SS-1). This triggers an inflammatory host immune response, which does not only weaken the enterocyte barrier function, but also helps STM to compete with the intestinal microbiota (2). While the T3SS-1 itself can already mediate adhesion (3), additional adhesive structures such as Fim fimbriae (4) or the giant non-fimbrial adhesin SiiE (5) are also critical for bacterial attachment, depending on the type of host cell. Transcriptional co-regulation of SPI-1 with the SiiE-encoding Salmonella pathogenicity island 4 (SPI-4) is the basis of this functional cooperation $(5,6)$

In line with previous findings on other polarized cells (7), it was recently shown that apical invasion of intestinal epithelial cells requires SiiE (8). SiiE likely functions as a lectin recognizing glycostructures with terminal $\mathrm{N}$-acetylglucosamine (GIcNAc) and/or a 2,3-linked sialic acid residues (9). The unique structural features of the $\sim 175 \mathrm{~nm}$ long $600 \mathrm{kDa}$ adhesin SiiE allow Salmonella to overcome the antiadhesive barrier function of the transmembrane, epithelial mucin MUC1, the extracellular domain of which is heavily decorated with O-linked glycans terminating in negatively charged sialic acids (8). SiiE comprises 53 bacterial immunoglobulin-like (Blg) domains that show distinct $\mathrm{Ca}^{2+}$ binding motifs crucial for its rigid tertiary structure $(10,11)$. SiiE is the only known substrate of the SPI-4-endoced T1SS and contains a complex C-terminal secretion signal (7). Based on similarity to other T1SS, e.g., the E. coli hemolysin system (12), secretion is likely achieved in a single step without a periplasmic intermediate. Considering these structural features, SiiE is thought to be permanently secreted into the extracellular space. A recent study showed that secreted SiiE suppressed the humoral immune response against Salmonella by reducing the number of IgG-secreting plasma cells in the bone marrow. Mechanistically, an $\mathrm{N}$-terminal region of $\mathrm{SiiE}$ with high similarity to laminin $\beta 1$ bound to $\beta 1$ integrin (CD29) on $\operatorname{lgG}^{+}$plasma cells, thereby preventing their interaction with laminin $\beta 1^{+} \mathrm{CXCL} 12^{+}$stromal cells which otherwise form 
a survival niche for plasma cells in the bone marrow (13). However, in line with its function as an adhesin, the protein was also found temporally retained on the surface of Salmonella $(7,8,14)$. Nonetheless, it is still unclear how the surface expression of SiiE and hence the switch between its function as adhesin vs. immunosuppressant is regulated.

Here, we show that the presence of aspartate (Asp) promotes the surface expression of SiiE and the adhesion of Salmonella, which turned out to be dependent on the Salmonella-specific methyl-accepting chemotaxis protein (MCP) CheM, an ortholog of E. coli Tar/MCP-II. Using mass spectrometry, CheM and other MCPs were identified as interaction partners of the SPI-4 encoded SiiA and SiiB. SiiAB are associated with the T1SS and form an inner membrane proton $\left(\mathrm{H}^{+}\right)$channel with similarities to the ExbB/TolQ and MotAB family $(15,16)$. Using a set of consecutive MCP deletion mutants, we found that invasion of polarized cells by STM was attenuated upon deletion of cheM. Binding of Asp to CheM usually triggers bacterial chemotaxis towards the attractant gradient (17). We discovered that the addition of a non-metabolizable aspartate analog, $\alpha$-methyl-D,L-aspartate (MeAsp), elevated host cell invasion by STM. Using mutants lacking downstream components of the chemotaxis phosphorelay or functional flagella, we observed that neither classical chemotaxis signaling nor bacterial motility contributed to the increased invasion. Instead, attractant-stimulation of CheM caused a shift from secreted to surface-retained and adhesion-competent SiiE. We therefore suggest that aspartate acts as microenvironmental cue that elicits the SPI-4-dependent adhesion to and invasion of polarized epithelial cells by Salmonella through a novel, non-canonical signaling pathway of the MCP CheM via SiiAB to the SPI-4 T1SS and SiiE. 


\section{RESULTS}

\section{SiiAB interact with CheM}

Our previous results suggested that SiiE-mediated adhesion depends on the function of the SPI-4 T1SSassociated SiiAB proton channel (15). We set out to identify protein interaction partners as potential regulators of SiiA and/ or SiiB. Epitope-tagged SiiA or SiiB was expressed from low copy-number plasmids and used as bait proteins to purify complexes after crosslinking. Composition of complexes was determined by liquid chromatography coupled to mass spectrometry (LC-MS/MS). As expected, both bait proteins were under the top five identified proteins (Fig 1A). Moreover, our data confirmed the previously found (15) interaction between SiiA and SiiB, since both proteins were identified as prey while using the other as bait. Interestingly, a set of MCPs (Aer, Trg, McpB, MсpC and CheM) was enriched in the SiiA complex while in the SiiB complex the MCP CheM was identified. MCPs act as sensors controlling flagellar movement towards attractants and away from repellants (18). In the following, we focused on CheM because the protein was previously implicated to have a role in Salmonella invasion of HeLa cells $(19,20)$ and appears to interact with both SiiA and SiiB. CheM is a Salmonella-specific MCP and an ortholog of E. coli Tar, but shares only $79 \%$ of sequence identity. Like known for Tar, the amino acid L-aspartate functions as an attractant stimulus for CheM while $\mathrm{Co}^{2+}$ and $\mathrm{Ni}^{2+}$ ions act as repellants $(17,21)$. However, in contrast to Tar, CheM does not respond to maltose (22).

To confirm the interactions between SiiAB and CheM identified by MS, we performed a bacterial twohybrid (B2H) assay which is based on the functional complementation of Bordetella pertussis adenylate cyclase (CyaA) from T25 and T18 fragments (23). The T25 fragment was fused to SiiA, SiiB, SiiD, SiiF or to a non-functional SiiA ${ }^{D 13 N}$ mutant (15), while the T18 fragment was fused to CheM. Blue colonies of the cyaA-deficient E. coli reporter strain BTH101 (24) indicated functional CyaA protein complementation and thus protein-protein interaction. We observed a strong interaction (dark blue colonies) between CheM-T18 and T25 fusions of SiiA and SiiA-D13N (Fig 1B). Moreover, high reporter activity was also observed when testing for the known dimerization of Tar/CheM (18) with co-expression of CheM-T18 and T25-CheM or CheM-T25 (Fig 1B). Lighter blue colonies were observed for the coexpression of CheM-T18 and SiiB-T25 or T25-SiiD showing $\beta$-galactosidase activity comparable to that of the positive control (Fig 1B). Furthermore, we performed co-immunopecipitation (co-IP) using epitope tagged proteins. When CheM-3×Flag was used as bait, both SiiA and SiiB were identified as prey proteins. Vice versa, using SiiB-3×Flag as bait, SiiA and epitope-tagged CheM-HA were detected as 
interaction partners (Fig 1C). Thus, using three independent approaches, we established that CheM interacts with both SiiA and SiiB while confirming the known SiiAB complex (15) and CheM dimerization (18).

\section{Role of MCPs for invasion of polarized MDCK}

Bacterial motility is required for efficient invasion of Salmonella into HeLa (25) and polarized Caco-2 cells (26). In a previously published study, we further assessed the impact of chemotaxis on invasion efficiency of non-polarized HeLa cells using sequential deletion of up to seven MCP genes (19). We found that loss of CheY or CheM led to an increase of Salmonella invasion (19) as observed earlier for smooth swimming mutants $(20,27)$. Because SPI-4 function was shown to play a role for adhesion to polarized cells only $(5,7,8)$, we aimed to investigate the role of individual MCPs on Salmonella invasion of polarized Madin-Carby Canine Kidney (MDCK) cells. Host cells were infected with STM wild-type (WT), a smooth swimming cheY mutant, $\Delta$ siiF (non-functional SPI-4 T1SS) and MCP mutant strains as described (19), followed by quantification of intracellular bacteria and subsequent normalization to STM WT. Interestingly, all MCP mutants missing the cheM gene exhibited reduced invasion in polarized MDCK cells. In contrast, elevated invasion rates were observed for the same cheM-lacking mutants when using non-polarized HeLa cells as described before (19) (Fig 2). While a smooth swimming $\Delta$ che $Y$ strain showed a 2-fold increased invasion rate in HeLa, the mutant was significantly attenuated in MDCK arguing for a role of chemotaxis for efficient invasion of polarized cells. In line with the known importance of SPI-4 for the adhesion to and invasion of MDCK cells (7), very few intracellular bacteria harboring an E627Q mutation within the Walker B motif of the SiiF ABC protein were detected (Fig 2). Thus, the type of infection model (non-polarized vs. polarized cells) determine the impact of CheM function on STM invasion which mirrors the differences seen for SPI-4 function (7).

\section{CheM attractant binding fosters Salmonella invasion of polarized cells}

To characterize a possible functional link of CheM to the SPI-4-encoded T1SS, we constructed two lowcopy-number plasmids that encode Salmonella cheM or, as a control, E. coli tar, each modified with a C-terminal 3×Flag-tag under control of the STM cheM promoter $\left(\mathrm{P}_{c h e M}\right)$. After introducing the plasmids in a mutant lacking all seven MCP genes $(\Delta 7 \mathrm{MCP})(19)$, Western blot demonstrated similar expression of both proteins with the cytosolic protein DnaK as loading control (Fig $3 A$ ). Next, $\triangle 7$ MCP was transformed with the empty vector control (pWSK29) and plasmids encoding for CheM (pCheM) or Tar (pTar) without epitope tag and these strains were further functionally characterized in swarming assays 
using soft agar plates. While the strain harboring pWSK29 did not swarm, pCheM and pTar conferred swarming ability to the mutant. However, compared to STM WT (> $5 \mathrm{~cm}$, not shown), both plasmidcomplemented $\Delta 7 \mathrm{MCP}$ showed a reduced swarming distance with $\sim 4 \mathrm{~cm}(\mathrm{pCheM})$ and $\sim 1 \mathrm{~cm}$ (pTar), respectively (Fig 3B). To test more specifically the ability to respond to CheM attractants, we performed a capillary assay as described by Adler (28) using MeAsp (29) (Fig 3C, left panel). Quantifying the bacteria within the fixed-volume capillary revealed significantly more cells in case of the pCheMcomplemented strain, compared not only to the vector control, but also compared to WT (Fig 3C, right panel).

We hypothesized that not the CheM protein itself, but rather CheM signaling elicited by the binding of CheM ligands (i.e. attractants) may have an impact on SPI-4 function and subsequently on invasion of polarized epithelial cells. Usually, attractant binding inactivates autokinase activity of MCP-coupled CheA, thus reducing phosphoryl transfer to the response regulators $\mathrm{CheY}$ and $\mathrm{CheB}$. While low CheY P results in counter-clockwise (CCW) flagellar rotation and straight swimming, receptor methylation is high due to low CheB P methylesterase activity (18). Therefore, STM WT and the $\triangle 7 \mathrm{MCP}$ mutant containing either the vector control, pCheM or pTar were tested for invasion of MDCK without attractant, in the presence of $10 \mathrm{mM}$ MeAsp or, as a control, $10 \mathrm{mM}$ of the non-metabolizable Tsr attractant a-aminoisobutyrate $(\mathrm{AiBu})(30,31)$. While AiBu had no or, in case of STM WT, even a detrimental effect on invasion, addition of MeAsp elevated invasion capability of WT and pCheM-complemented $\triangle 7 \mathrm{MCP}$ (Fig 3D). The pCheM vector partially complemented the invasion defect of the $\Delta 7 \mathrm{MCP}$ mutant in the absence of attractant or with addition of $\mathrm{AiBu}$, while the strains carrying pTar or the vector control were attenuated for invasion regardless of attractant supplementation (Fig 3D).

To verify our findings obtained with MDCK cells, we employed HT29-MTX cells $(8,32)$ as an alternative infection model. In contrast to non-polarized 1-day cultures (Fig S1A, upper panel), polarized monolayers with significant amounts of mucus were formed after 21 days of culture (Fig S1A, lower panel). Similar to HeLa cells (7), Salmonella invasion of non-polarized HT29-MTX cells required T3SS-1 but was independent of SPI-4 (Fig S1B). Invasion of polarized HT29-MTX cells, however, was strongly dependent on an intact SPI-4 locus (Fig 3E) as observed before (8). In close accordance with the MDCK data, elevated invasion of HT29-MTX cells was observed for WT and $\triangle 7$ MCP [pCheM] in the presence of MeAsp (Fig 3E). However, pCheM was able to complement the $\triangle 7 \mathrm{MCP}$ mutant to the level of WT STM without attractant or with $10 \mathrm{mM} \mathrm{AiBu}$. In contrast, low invasion rates were observed for cells without CheM (Fig 3E). 
Taken together, using two infection models based on polarized cells, we observed a stimulating effect of the CheM ligand MeAsp on Salmonella invasion. The phenotype was specifically dependent on the presence of CheM. No increase in invasion was seen in strains only expressing E. coli Tar or with addition of the Tsr ligand AiBu.

\section{Augmented invasion after CheM stimulation is independent of motility and chemotaxis}

Bacterial motility and chemotaxis towards energy sources was shown to be required for Salmonella virulence in vivo (33-35). Because our data also suggest a promoting effect of chemotaxis for invasion of polarized cells, we set out to characterize the role of motility and the chemotaxis phosphorelay pathway for the observed phenotype in more detail. We generated a non-motile mutant lacking the flagella-specific ATPase flil and employed, besides the cheY-deficient strain, a mutant lacking the histidine autokinase CheA. Together with MCPs and the coupling protein CheW, CheA dimers form a ternary complex that is the minimum requirement for chemosensing $(18,36)$. The che $A$ and cheY mutations were further combined with the $\triangle 7 \mathrm{MCP}$ mutant. These mutant strains were all attenuated for invasion of MDCK. Interestingly, the non-motile $\Delta$ flil and the two 8-fold mutants ( $\Delta 7 \mathrm{MCP}$ plus $\Delta c h e A$ and $\Delta 7 \mathrm{MCP}$ plus $\Delta$ cheY) exhibited an even stronger phenotype with almost no invasion detectable (Fig 4A). While significantly more intracellular WT STM bacteria were found when grown in the presence of MeAsp, the mutants responded neither to this attractant nor to AiBu (Fig 4A). Motile bacteria exhibit an invasion advantage due to near surface swimming and thus higher probability to encounter a host cell (25). To compensate for the lack of motility, we used centrifugation to bring bacteria into close proximity to the host cells, which allows investigating bacterial invasion following adhesion despite the absence of bacterial motility. Under these conditions, the $\Delta$ flil mutant behaved like WT with significantly increased invasion in the presence of MeAsp (Fig 4B). The invasion capability of the $\triangle 7 \mathrm{MCP}$ mutant was not altered by centrifugation. While $\Delta c h e A$ and $\Delta c h e Y$ mutants behaved similar to WT bacteria without attractant or with $\mathrm{AiBu}$, they showed vastly increased invasion rates when MeAsp was added (Fig 4B). In contrast, Salmonella with a cheA or cheY mutation and simultaneous deletion of all MCPs ( $\Delta$ cheA $\Delta 7 \mathrm{MCP}$ or $\Delta c h e Y \Delta 7 \mathrm{MCP}$ ) lost the responsiveness to MeAsp and the ability to invade host cells. Thus, MeAsp fosters Salmonella invasion in a CheM-dependent manner, but this process is independent of bacterial motility and the chemotaxis phosphorelay pathway. 
Salmonella invasion after MeAsp stimulation. Instead, the pronounced phenotype in conjunction with polarized cells and the identification of CheM as a SiiAB interaction partner argues for CheM as a regulator of SPI-4 dependent adhesion. Previous studies suggested that SPI-4 adhesion capability is determined by the amount and/ or binding strength of surface-localized SiiE $(7,14)$. Therefore, mechanisms regulating SiiE surface expression might account for SPI-4 dependent adhesion. To test whether attractant binding to CheM affects localization of SiiE, we quantified the amounts of surface-retained and secreted SiiE adhesin after $3.5 \mathrm{~h}$ of growth (late logarithmic phase) with or without addition of MeAsp. Bacteria-associated SiiE was quantified in a dot blot assay using a SiiE-specific antibody and normalization to the LPS signal. Upon addition of MeAsp, elevated amounts of retained SiiE were detected for WT STM and the $\triangle 7$ MCP mutant carrying pCheM (Fig $5 \mathrm{~A}$ ). In contrast, no upregulation of surface-localized SiiE in response to MeAsp was observed for the $\Delta 7$ MCP mutant harboring the empty vector or for the $\Delta$ siiE mutant which served as negative control (Fig $5 \mathrm{~A}$ ). Quantification of secreted SiiE using a specific ELISA (6) revealed an inhibitory effect of CheM attractant binding reciprocal to SiiE surface localization. MeAsp inhibited SiiE secretion of WT and $\Delta 7$ MCP [pCheM] to the level of the $\Delta$ siiE control. Interestingly, compared to WT STM, almost 2-fold more SiiE was secreted from the $\triangle 7 \mathrm{MCP}$ strain harboring the empty vector control (Fig $5 \mathrm{~B}$ ).

Our findings support a model where the interplay of CheM with the SPI-4 components SiiAB regulates SiiE localization. Attractant binding by CheM resulted in more surface-localized SiiE. To test whether indeed surface-retained SiiE can function as an adhesin, we combined competitive index experiments with a screen for ligand expression using immunomagnetic particles (SIMPLE) (37) (Fig 6A). The test and reference strains harboring different antibiotic resistance cassettes were mixed equally and an aliquot was plated on appropriate selective media to verify the proportion of the two strains. Subsequently, $\alpha$-SiiE antibodies and magnetic protein A beads were added to the mixture. SiiE-positive bacteria were enriched through magnetic separation of beads coated with antibodies that have bound their antigen. Finally, the proportion of test and reference strain was determined through parallel plating (Fig 6A). Using STM WT as test strain and $\Delta$ siiE as reference, we achieved $\sim 8$-fold enrichment using this assay. As expected, there was no effect of MeAsp on enrichment of STM WT over $\Delta$ siiE (Fig 6B). When the $\triangle 7$ MCP strain was used as reference, WT STM was $\sim 3$-fold enriched in the presence of MeAsp, while no enrichment was seen without attractant (Fig 6B). These results demonstrate that addition of MeAsp enhanced the localization of SiiE on the surface of Salmonella in a MCP-dependent manner. 


\section{DISCUSSION}

241 In the present study, we identified the MCP CheM as a novel SiiAB interaction partner and the binding

242 of attractants to CheM as a positive regulator of SPI-4 dependent adhesion. We found that straight 243 swimming cheA or cheY mutants, which resemble an attractant-bound "always off" state of the MCPs 244 and are incapable of phosphoryl transfer, were attenuated for invasion in polarized cells. In contrast, 245 straight swimming Salmonella showed a higher probability to invade other cell types $(19,25)$. Recently, the Salmonella MCP McpC was shown to promote a straight swimming phenotype that was dependent on the SPI-1 transcription factor HilD (38). When we bypassed the impact of motility and chemotaxis on bacteria-host cell interaction by centrifugation, addition of the CheM attractant MeAsp was still able to enhance invasion of polarized cells. This was particularly remarkable for the non-motile $\Delta$ flil strain, which by itself rules out any involvement of the "classical" chemotaxis-motility pathway. In the absence of MeAsp, centrifugation of $\Delta c h e A$ and $\Delta c h e Y$ mutants led to invasion rates comparable to WT. Strikingly, in the presence of the CheM attractant, these strains were hyperinvasive ( 20-25-fold of WT). In contrast, invasion capability was completely abolished with additional deletion of all 7 MCP genes. These observations cannot be explained with the chemotaxis phosphorelay signaling (18). Therefore, we propose a novel, non-canonical signal transduction from the MCP to SPI-4 encoded proteins resulting in increased adhesion and subsequent bacterial invasion. Links between chemotaxis and bacterial virulence functions are not unprecedented. In Pseudomonas aeruginosa, the putative MCP encoded by $P A 2573$ regulates genes involved in virulence and antibiotic resistance and the soluble chemoreceptor McpB was shown to be important for virulence in several infection models $(39,40)$. In Cronobacter sakazakii, a plasmid-encoded MCP was reported to have an impact on adhesion, invasion, motility and biofilm formation (41). The MCPs Tcpl and AcfB of Vibrio cholerae were shown to be important for infant mouse colonization (42). For plant pathogenic bacteria such as Agrobacterium tumefaciens or Xanthomonas oryzae pv. oryzae, many chemoattractants can also act as virulence inducers $(43,44)$. However, in all these examples chemotaxis signaling is mechanistically linked to virulence through transfer of phosphoryl groups to alternative response regulators resulting in a virulence-specific transcriptional response (36).

The transduction of the CheM attractant signal is presumably based on the identified interaction of the MCP with the SPI-4 T1SS-associated SiiAB proton channel. It is tempting to postulate a regulation of SiiAB proton flux through direct interaction with attractant-bound CheM (Fig 7). The peptidoglycan (PG) 
binding domain of MotB was shown to function as a plug sealing the proton channel. Upon association with the flagellar motor complex, the MotB domain is shifted through PG binding and thereby enables proton flux and energy conversion of the system (45). Similarly, the SiiA PG binding domain (16) could be displaced from the SiiAB proton channel through interaction with attractant-bound CheM. In orthologous E. coli Tar dimers, Asp binding induces a piston-like movement of one alpha helix within the sensory domain. This movement is amplified in the cytosolic HAMP domains and finally transmitted to the hairpin tip bundle controlling CheA autokinase activity (18). In our model, the structural changes within the CheM ligand binding domain, and perhaps other portions of the molecule, would change the molecular interface between CheM and the SiiAB channel, thus enabling PG binding of SiiA and proton flux. The energy harvested from the transmembrane $\mathrm{H}^{+}$gradient would then be transferred to the SPI-4 encoded T1SS by means of an energy-rich conformation resulting in retention of the SiiE molecule (Fig 7). Such energy transfer has been described for the SiiAB homologs ExbBD and TolQR inducing conformational changes in TonB and TolA, respectively $(46,47)$. Interestingly, in vitro studies with the isolated periplasmic domain of SiiA showed a pH-dependency of PG binding activity. SiiA PG-binding was observed at $\mathrm{pH}$ 5.8-6.2, but not between $\mathrm{pH} 6.7$ and $\mathrm{pH} 8.0$ (16). Here, slightly acidic $\mathrm{pH}$ as found in some parts of the gut could serve as another environmental signal to regulate the adhesion capacity of SPI-4. Alternatively, the observed pH-dependent PG binding could function as a proxy to ensure sufficient energization by detecting periplasmic protons contributing to the proton motif force (PMF). According to our model (Fig 7), Salmonella utilizes Asp as an environmental cue to control SiiE surface expression. Aspartate and other free amino acids are generated from oligopeptides originating from food through the activity of peptidases at the apical side of polarized enterocytes (48). The bulk of this amino acid liberation, and subsequent absorption, takes place in the proximal jejunum and is usually complete at the terminal ileum $(48,49)$. Although there is extensive catabolism of enteral Asp by enterocytes (50) and gut bacteria (51), the microbiota also releases free Asp through peptide degradation (52). Recently, Asp was found to contribute to initial murine gut colonization of STM by enabling hydrogen/fumaratedependent anaerobic respiration. Aspartate is taken up in exchange of succinate by the high-affinity antiporter DcuABC and converted to the alternative electron acceptor fumarate (53). Therefore, the availability of Asp within the small intestine not only enables bacterial expansion in competition to the intestinal microbiota, but also contributes, amongst other environmental stimuli, to precise spatiotemporal control of bacterial adhesion to polarized epithelial cells. 
bioRxiv preprint doi: https://doi.org/10.1101/2021.05.14.443827; this version posted May 15, 2021. The copyright holder for this preprint (which was not certified by peer review) is the author/funder. All rights reserved. No reuse allowed without permission.

301 Asp was identified as an attractant of CheM that elicited a change in the localization of the giant SPI-4encoded adhesin SiiE of Salmonella: in the absence of Asp, SiiE was primarily secreted, whereas in the presence of Asp, SiiE was retained on the bacterial surface. Surface-bound, but not secreted SiiE

304 functions as an adhesin. Thus, the CheM attractant L-aspartate acts as positive regulator of SPI-4dependent adhesion to polarized cells. Although CheM directly interacts with the SPI-4 encoded SiiAB proton channel, the exact molecular mechanisms of signal transductions and adhesin retention remain to be characterized. 


\section{MATERIALS AND METHODS}

\section{Bacterial strains and plasmids}

All strains used are listed in Table S1. Bacteria were routinely grown in LB media supplemented with $50 \mu \mathrm{g} / \mathrm{mL}$ carbenicillin (Cb) (Carl Roth, Mannheim, Germany), $25 \mu \mathrm{g} / \mathrm{mL}$ kanamycin (Km) (Carl Roth), $10 \mu \mathrm{g} / \mathrm{mL}$ chloramphenicol (Cm) (Carl Roth), $50 \mathrm{ng} / \mathrm{mL}$ anhydrotetracycline (AHT) (\# 37919 SigmaAldrich, Schnelldorf, Germany), $10 \mathrm{mM}$ a-aminoisobutyrate (AiBu) (\#850993 Sigma-Aldrich) or $10 \mathrm{mM}$ a-methyl-D, L-aspartate (MeAsp) (\#M6001 Sigma-Aldrich), if required. For details on the construction of mutant strains and plasmids, the reader is referred to the supplementary information. Tables S2 and S3 give an overview of all the plasmids and primers used in this study, respectively.

\section{Protein-protein interaction assays}

Bacterial two hybrid (B2H) assays were essentially carried out as described before (15). Briefly, E. coli reporter strain BTH101 was co-transformed with plasmids encoding for protein fusions with the T18 and T25 fragments of Bordetella pertussis CyaA. Transformants were spread on LB plates containing $25 \mu \mathrm{g} / \mathrm{mL}$ kanamycin, $100 \mu \mathrm{g} / \mathrm{mL}$ carbenicillin, $100 \mu \mathrm{M}$ IPTG (Thermo Scientific, St. Leon-Rot, Germany) and $40 \mu \mathrm{g} / \mathrm{mL}$ X-Gal (5-bromo-4-chloro-3-indolyl- $\beta$-D-galactopyranoside; Thermo Scientific). Plates were incubated at room temperature for $48-72 \mathrm{~h}$, and blue colonies indicated protein interaction resulting in functional CyaA-complementation.

For co-immunoprecipitation (co-IP), STM were either transformed with pWRG868 (CheM-3×Flag) or co-

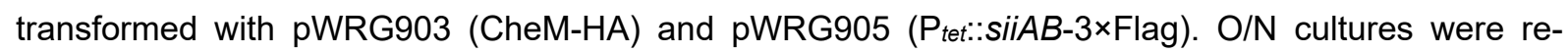
inoculated 1:31 into $500 \mathrm{~mL}$ fresh medium and grown with aeration for $3.5 \mathrm{~h}$. The expression of SiiAB$3 \times$ Flag was induced for $2 \mathrm{~h}$ with AHT. Cells were pelleted $(8,000 \times \mathrm{g}, 10 \mathrm{~min}$, room temperature $(\mathrm{RT}))$, re-suspended in $250 \mathrm{~mL}$ of pre-warmed MEM medium (Capricorn Scientific, Ebsdorfergrund, Germany) and allowed to grow for additional 30 min. Proteins were crosslinked with addition of $0.5 \%(\mathrm{w} / \mathrm{v})$ paraformaldehyde (\#43368 Alfa Aesar, Heysham, UK). After $15 \mathrm{~min}$, the reaction was quenched with $0.125 \mathrm{M}$ glycine. Cells were washed thrice with ice-cold MEM medium and then stored at $-20 \mathrm{C}$. Pellets were re-suspended in $10 \mathrm{~mL}$ PBS supplemented with $1 \% n$-dodecyl $\beta$-D-maltoside (\# A0819, AppliChem, Darmstadt, Germany), 1× EDTA-free Halt protease inhibitor cocktail (\#87785, Thermo Fisher Scientific, Karlsruhe, Germany), $0.5 \mathrm{mM} \mathrm{MgSO} 4$ and $5 \mu \mathrm{L}$ TURBO DNase (Ambion). Subsequently, cells were lysed through a French pressure cell (EmulsiFlex-C3, Avestin, Mannheim, 
Germany) and debris was removed by low speed centrifugation $\left(11,000 \times \mathrm{g}, 20 \mathrm{~min}, 4^{\circ} \mathrm{C}\right)$. The protein extract, containing either SiiB-3×Flag or CheM-3×Flag, was further cleared by additional centrifugation $\left(20,000 \times \mathrm{g}, 15 \mathrm{~min}, 4^{\circ} \mathrm{C}\right)$. The protein concentration was measured by BCA assay (\#23225, Thermo Fisher) and similar protein amounts were used for co-IP of all samples. Immunoprecipitation of $3 \times$ Flagtagged proteins was performed using a-FLAG M2 affinity gel (Sigma-Aldrich) following the manufacturer's recommendations. Therefore, $100 \mu \mathrm{L}$ of the gel suspension (50 $\mu \mathrm{L}$ of packed gel volume) were washed $3 \times$ with $1 \mathrm{~mL}$ of PBS and subsequently added to each sample. Protein binding was allowed over night at $4^{\circ} \mathrm{C}$. After three further washing steps, bound proteins were eluted from the beads with addition of $50 \mu \mathrm{L}$ reducing sample buffer (Carl Roth) and heating for $15 \mathrm{~min}$ to $98^{\circ} \mathrm{C}$.

\section{Western blot}

Aliquots of protein samples were mixed with reducing sample buffer (Carl Roth) to a final concentration of $1 \times$. After heating to $98^{\circ} \mathrm{C}$ for $15 \mathrm{~min}, 10 \mu \mathrm{L}$ of each sample was analyzed by SDS-PAGE electrophoresis (ProSieve, Lonza, Cologne, Germany) and subsequent Western blot (Bio-Rad, Munich, Germany) on a polyvinylidene difluoride membrane (Thermo Fisher). Membranes were probed with antibodies against DnaK (clone 8E2/2, Enzo Life Science, Lörrach, Germany), SiiA, SiiB (15), HA (clone 3F10, Roche, Mannheim, Germany) or Flag (M2, Sigma-Aldrich) and appropriate horseradish-coupled secondary antibodies (Dianova, Hamburg, Germany).

\section{Mass spectrometry}

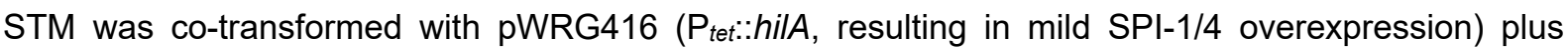
pWRG461 (siiA-3×Flag) or with pWRG416 plus pWRG462 (siiAB-3×Flag). Protein complexes were purified with a-FLAG M2 affinity gel (Sigma-Aldrich) as described for co-IP. After washing, bead-bound proteins were eluted twice with $450 \mu \mathrm{L}$ of $0.1 \mathrm{M}$ glycine $\mathrm{pH} 3.5$ and $180 \mu \mathrm{L}$ of $0.5 \mathrm{M}$ Tris- $\mathrm{HCl} \mathrm{pH} 7.4$, $1.5 \mathrm{M} \mathrm{NaCl}$ was added for neutralization. To precipitate the proteins, trichloracetic acid was added to a final concentration of $10 \%$ and the samples were incubated $\mathrm{O} / \mathrm{N}$ at $4^{\circ} \mathrm{C}$. After centrifugation $(20,000 \times \mathrm{g}$, $45 \mathrm{~min}, 4^{\circ} \mathrm{C}$ ), the pellets were washed twice with ice-cold acetone. The air-dried pellet was finally suspended in $100 \mu \mathrm{L}$ fresh $50 \mathrm{mM} \mathrm{NH}_{4} \mathrm{HCO}_{3}, \mathrm{pH} 7.8$. The samples were subjected to tryptic digestion and the resulting peptide mixtures were analyzed by nano-ESI-LC-MS/MS (Thermo Scientific LTQ Orbitrap). Proteins were identified using Mascot (Matrix Science, London, UK) based on a custom proteome file of S. Typhimurium strain ATCC 14028s. Spectral counts were extracted using Scaffold 

applying upper quartile normalization and interquartile range filtering. Data is summarized in Table S4.

\section{Cell culture and infection}

HT29-MTX human colonic epithelial cells (kind gift of G. Grassl, Hannover, Germany) were cultured in DMEM medium (high glucose, stable glutamine, sodium pyruvate) (Biowest, Nuaillé, France) supplemented with 10\% FCS and non-essential amino acids (Biowest). HeLa cells (ATCC CCL-2, LGC Standards, Wesel, Germany) were grown in DMEM (Biowest) supplemented with 10\% FCS, sodium pyruvate and $2 \mathrm{mM}$ GlutaMax (Thermo Fisher) and MDCK cells (subclone Pf, Department of Nephroplogy, FAU Erlangen-Nürnberg) were kept in MEM medium (Biowest) supplemented with 10\% FCS, 2 mM Glutamax (Thermo Fisher) and non-essential amino acids (Biowest). To each medium $100 \mathrm{U} / \mathrm{mL}$ penicillin and $100 \mu \mathrm{g} / \mathrm{mL}$ streptomycin (Biowest) were added. Cultures were incubated at $37^{\circ} \mathrm{C}$ in a humidified $5 \%(\mathrm{v} / \mathrm{v}) \mathrm{CO}_{2}$ atmosphere. For invasion assays, $\mathrm{HT} 29-\mathrm{MTX}$ cells were seeded in 24-well culture plates (\#662160, Cellstar, Greiner Bio-One, Frickenhausen, Germany) at a density of $4 \times 10^{4}$ cells per well 21 days prior infection. MDCK and HeLa cells were seeded in 96-well plates (\#655180, Greiner Bio-One) at a density of $8 \times 10^{4}$ or $6 \times 10^{3}$ per well, respectively. MDCK cells were allowed to polarize for 10-11 days. The culture medium was changed every other day and medium without antibiotics was used for the last medium change.

Gentamicin protection assays were essentially carried out as described previously [7]. Briefly, bacterial overnight $(\mathrm{O} / \mathrm{N})$ cultures grown in LB supplemented with appropriate antibiotics were re-inoculated 1:31 in fresh medium and grown aerobically for another $3.5 \mathrm{~h}$. An inoculum corresponding to a multiplicity of infection (MOI) of 10 (HeLa) or 25 (MDCK, HT29-MTX) was prepared in MEM/DMEM and used to infect the cells for $25 \mathrm{~min}$. After the cells had been washed thrice with PBS, MEM/DMEM containing $100 \mu \mathrm{g} / \mathrm{mL}$ gentamicin was applied to each well to kill remaining extracellular bacteria. After $1 \mathrm{~h}$ of incubation, the cell layers were washed again with PBS and then lysed for 10 min with PBS containing $1 \%$ Elugent of invasive bacteria was calculated and subsequently normalized to WT.

\section{Swarming assay}


$\mathrm{NaCl}, 0.5 \%$ agar) as described before (19). Briefly, a small amount $(0.2 \mu \mathrm{L})$ of bacterial $\mathrm{O} / \mathrm{N}$ cultures was applied onto the center of LB soft agar plate and incubated for six hours at $37^{\circ} \mathrm{C}$. The diameters of the swarm colonies were measured and the plates were photographed.

\section{Capillary assay}

Capillary assays were essentially performed as described before (28) with the following modifications: An U-shaped dam created from a piece of modelling clay and parafilm was mounted onto a microscopy slide. The chamber thus created was sealed with a cover slip and filled with $500 \mu \mathrm{L}$ of a $3.5 \mathrm{~h}$ bacterial sub-culture. A $1 \mu \mathrm{L}$ capillary (BLAUBRAND intraEND, Brand, Wertheim, Germany) was heat-sealed at one end and then filled with a $100 \mathrm{mM}$ MeAsp solution. The capillary prepared in this way was immersed in the chamber for $1 \mathrm{~h}$ at $37^{\circ} \mathrm{C}$. The capillary was then rinsed, the sealed end broken off and the capillary contents was emptied using a pipetting aid (Brand). Serial dilutions were plated and CFUs determined

\section{ELISA}

Antisera were raised in rabbits against the recombinant C-terminal moiety of SiiE (7). For detection of SiiE, culture supernatants were filter-sterilized $(0.45 \mu \mathrm{m}$ syringe filters, Corning Life Sciences, Amsterdam, Netherlands), and aliquots of $50 \mu \mathrm{L}$ were directly applied to 96-well Nunc MultiSorp microtiter plates (\#467340 Thermo Fisher) overnight at $4^{\circ} \mathrm{C}$ in a humid chamber. The plates were washed three times with $200 \mu \mathrm{L} /$ well of PBS supplemented with $0.05 \%$ Tween20 (PBS-Tween), and the rabbit anti-glutathione S-transferase(GST)-SiiE-C detection antibody diluted 1:1,000 in PBS plus 10\% inactivated FCS (PBS-FCS) was applied for $2 \mathrm{~h}$ at RT. After five washes with PBS-Tween, $100 \mu \mathrm{L}$ of the anti-rabbit horseradish peroxidase-coupled secondary antibody diluted 1:2,500 in PBS-FCS was added to each well for 30 min at RT. The wells were washed again seven times with PBS-Tween, and $50 \mu \mathrm{L}$ of enzyme-linked immunosorbent assay (ELISA) horseradish peroxidase substrate (\#555214, Becton Dickinson, Heidelberg, Germany) was added. After incubation in the dark at RT for 8 to 15 min, the reaction was stopped by the addition of $25 \mu \mathrm{L} /$ well $0.5 \mathrm{M} \mathrm{H}_{2} \mathrm{SO}_{4}$ and $\mathrm{A}_{450}$ was measured using a M1000 plate reader (Tecan, Männedorf, Switzerland).

\section{Dot Blot}

Bacterial strains were diluted 1:31 in LB from $\mathrm{O} / \mathrm{N}$ cultures and grown at $37^{\circ} \mathrm{C}$ for $3.5 \mathrm{~h}$. Aliquots of $1 \mathrm{~mL}$ additional washing step with sterile $\mathrm{LB}$, bacterial suspensions were adjusted to $\mathrm{OD}_{600}=1$ in $500 \mu \mathrm{L}$ of $3 \%$ 
PFA in PBS. After fixation of bacterial cells for $15 \mathrm{~min}$ at RT, cells were pelleted $(10,000 \times \mathrm{g}, 5 \mathrm{~min} ., \mathrm{RT})$ and re-suspended in $500 \mu \mathrm{L}$ PBS. Five microliters of bacterial suspensions were spotted on nitrocellulose membrane pieces, set in a black 24-well plate, which have been pre-wetted with PBS and dried again before adding bacteria. After drying of the spots, membranes were blocked with $5 \%$ dry milk powder and $3 \% \mathrm{BSA}$ in PBS/T (PBS + 0.1\% Tween20) for at least $30 \mathrm{~min}$. For detection of SiiE on the bacterial surface, antiserum against the C-terminal moiety of SiiE was diluted 1:5,000 in blocking solution and applied to the membrane. LPS was detected using antiserum against Salmonella O-antigen (Becton Dickinson) at the same dilution. After incubation $\mathrm{O} / \mathrm{N}$ at $4^{\circ} \mathrm{C}$, membranes were washed thrice with PBS/T and HRP-linked secondary antibody was added in a 1:50,000 dilution in PBS/T. After three additional washing steps with PBS/T, membranes were rinsed in PBS, substrate for HRP was applied and signals were quantified using a Tecan M1000 plate reader in luminescence mode.

\section{SIMPLE Assay}

A screen for ligand expression using immunomagnetic particles (SIMPLE) assay was carried out as described by Nuccio et al. (37) with the following modifications. Salmonella strains were sub-cultured $1: 31$ from $\mathrm{O} / \mathrm{N}$ for $3.5 \mathrm{~h}$ at $37^{\circ} \mathrm{C}$ and adjusted to $\mathrm{OD}_{600}=2$ in fresh PB buffer $(=\mathrm{TN}$ buffer $(0.1 \mathrm{M}$ Tris- $\mathrm{HCl}$ $\mathrm{pH} 7.5,0.15 \mathrm{M} \mathrm{NaCl}$ ) plus $1 \%$ casein). Strains carried either plasmid pWSK29 or derivatives to exhibit carbenicillin resistance or plasmid pWSK129 for a kanamycin resistance. The strains were mixed at a ratio of 1:1 and bacteria were then pre-incubated with $\alpha$-SiiE antibody or pre-immune serum in $650 \mu \mathrm{L}$ PB-buffer for $1 \mathrm{~h}$ at RT with head-over-head rotation. Then, $100 \mu \mathrm{L}$ of washed magnetic beads (BioMag Protein A, Qiagen, Hilden, Germany) resuspended in $100 \mu \mathrm{L}$ TN-buffer were added to each sample (total volume of $750 \mu \mathrm{L}$ ) and incubated for two additional hours. After three washing steps with $750 \mu \mathrm{L}$ TN buffer, beads were suspended in $1 \mathrm{~mL}$ PBS. Serial dilutions of all probes were plated in parallel on $\mathrm{MH}$ plates containing carbenicillin or kanamycin, CFU were determined and enrichment based on the competitive index and normalization to pre-immune serum controls was calculated.

\section{Acknowledgements}

We thank Guntram Grassl (Hannover, Germany) for providing cell line HT29-MTX. This work was funded by grants of the German research foundation (www.DFG.de) to Y.A.M. (MU 1477 9/2), M.H. (HE 1964 13/2) and R.G.G. (GE 2533 2/2). 
1. I. Behlau, S. I. Miller, A PhoP-repressed gene promotes Salmonella typhimurium invasion of epithelial cells. J Bacteriol 175, 4475-4484 (1993). B. Stecher et al., Salmonella enterica serovar Typhimurium exploits inflammation to compete with the intestinal microbiota. PLoS Biol 5, 2177-2189 (2007).

3. M. Lara-Tejero, J. E. Galán, Salmonella enterica serovar typhimurium pathogenicity island 1encoded type III secretion system translocases mediate intimate attachment to nonphagocytic cells. Infect Immun 77, 2635-2642 (2009).

4. B. Misselwitz et al., Salmonella enterica serovar Typhimurium binds to HeLa cells via Fimmediated reversible adhesion and irreversible type three secretion system 1-mediated docking. Infect Immun 79, 330-341 (2011).

5. R. G. Gerlach et al., Cooperation of Salmonella pathogenicity islands 1 and 4 is required to breach epithelial barriers. Cell Microbiol 10, 2364-2376 (2008).

6. R. G. Gerlach, D. Jäckel, N. Geymeier, M. Hensel, Salmonella pathogenicity island 4-mediated adhesion is coregulated with invasion genes in Salmonella enterica. Infect Immun 75, 46974709 (2007).

7. R. G. Gerlach et al., Salmonella Pathogenicity Island 4 encodes a giant non-fimbrial adhesin and the cognate type 1 secretion system. Cell Microbiol 9, 1834-1850 (2007).

8. X. Li et al., MUC1 is a receptor for the Salmonella SiiE adhesin that enables apical invasion into enterocytes. PLoS Pathog 15, e1007566 (2019).

9. C. Wagner, B. Barlag, R. G. Gerlach, J. Deiwick, M. Hensel, The Salmonella enterica giant adhesin SiiE binds to polarized epithelial cells in a lectin-like manner. Cell Microbiol 16, 962975 (2014).

10. M. H. Griessl et al., Structural insight into the giant $\mathrm{Ca}^{2+}$-binding adhesin SiiE: implications for the adhesion of Salmonella enterica to polarized epithelial cells. Structure 21, 741-752 (2013).

11. B. Peters et al., Structural and functional dissection reveals distinct roles of $\mathrm{Ca}^{2+}$-binding sites in the giant adhesin SiiE of Salmonella enterica. PLoS Pathog 13, e1006418 (2017).

12. S. Thomas, I. B. Holland, L. Schmitt, The Type 1 secretion pathway - The hemolysin system and beyond. Biochimica et biophysica acta 10.1016/j.bbamcr.2013.09.017 (2013).

13. C. Männe et al., Salmonella SiiE prevents an efficient humoral immune memory by interfering with $\mathrm{IgG}^{+}$plasma cell persistence in the bone marrow. Proc Natl Acad Sci U S A 116, 74257430 (2019).

14. C. Wagner et al., Functional dissection of SiiE, a giant non-fimbrial adhesin of Salmonella enterica. Cell Microbiol 13, 1286-1301 (2011).

15. T. Wille et al., SiiA and SiiB are novel type I secretion system subunits controlling SPI4-mediated adhesion of Salmonella enterica. Cell Microbiol 16, 161-178 (2014).

16. P. Kirchweger et al., Structural and functional characterization of SiiA, an auxiliary protein from the SPI4-encoded type 1 secretion system from Salmonella enterica. Mol Microbiol 112, 1403$1422(2019)$.

17. Y. Blat, M. Eisenbach, Tar-dependent and -independent pattern formation by Salmonella typhimurium. J Bacteriol 177, 1683-1691 (1995).

18. J. S. Parkinson, G. L. Hazelbauer, J. J. Falke, Signaling and sensory adaptation in Escherichia coli chemoreceptors: 2015 update. Trends Microbiol 23, 257-266 (2015).

19. S. Hoffmann, C. Schmidt, S. Walter, J. K. Bender, R. G. Gerlach, Scarless deletion of up to seven methyl-accepting chemotaxis genes with an optimized method highlights key function of CheM in Salmonella Typhimurium. PLoS One 12, e0172630 (2017).

20. B. D. Jones, C. A. Lee, S. Falkow, Invasion by Salmonella typhimurium is affected by the direction of flagellar rotation. Infect Immun 60, 2475-2480 (1992).

21. A. F. Kolodziej, T. Tan, D. E. Koshland, Jr., Producing positive, negative, and no cooperativity by mutations at a single residue located at the subunit interface in the aspartate receptor of Salmonella typhimurium. Biochemistry 35, 14782-14792 (1996).

22. T. Mizuno, N. Mutoh, S. M. Panasenko, Y. Imae, Acquisition of maltose chemotaxis in Salmonella typhimurium by the introduction of the Escherichia coli chemosensory transducer gene. J Bacteriol 165, 890-895 (1986).

23. G. Karimova, J. Pidoux, A. Ullmann, D. Ladant, A bacterial two-hybrid system based on a reconstituted signal transduction pathway. Proc Natl Acad Sci U S A 95, 5752-5756 (1998).

24. G. Karimova, A. Ullmann, D. Ladant, A bacterial two-hybrid system that exploits a cAMP signaling cascade in Escherichia coli. Methods Enzymol 328, 59-73 (2000).

25. B. Misselwitz et al., Near surface swimming of Salmonella Typhimurium explains target-site 
selection and cooperative invasion. PLoS Pathog 8, e1002810 (2012).

26. F. J. Van Asten, H. G. Hendriks, J. F. Koninkx, B. A. Van der Zeijst, W. Gaastra, Inactivation of the flagellin gene of Salmonella enterica serotype Enteritidis strongly reduces invasion into differentiated Caco-2 cells. FEMS microbiology letters 185, 175-179 (2000).

27. T. Khoramian-Falsafi, S. Harayama, K. Kutsukake, J. C. Pechere, Effect of motility and chemotaxis on the invasion of Salmonella typhimurium into HeLa cells. Microb Pathog 9, 47-53 (1990).

28. J. Adler, A method for measuring chemotaxis and use of the method to determine optimum conditions for chemotaxis by Escherichia coli. J Gen Microbiol 74, 77-91 (1973).

29. R. Mesibov, J. Adler, Chemotaxis toward amino acids in Escherichia coli. J Bacteriol 112, 315326 (1972).

30. S. Neumann, C. H. Hansen, N. S. Wingreen, V. Sourjik, Differences in signalling by directly and indirectly binding ligands in bacterial chemotaxis. EMBO J 29, 3484-3495 (2010).

31. M. L. Hedblom, J. Adler, Genetic and biochemical properties of Escherichia coli mutants with defects in serine chemotaxis. J Bacteriol 144, 1048-1060 (1980).

32. T. Lesuffleur, A. Barbat, E. Dussaulx, A. Zweibaum, Growth adaptation to methotrexate of HT29 human colon carcinoma cells is associated with their ability to differentiate into columnar absorptive and mucus-secreting cells. Cancer Res 50, 6334-6343 (1990).

33. F. Rivera-Chávez et al., Energy Taxis toward Host-Derived Nitrate Supports a Salmonella Pathogenicity Island 1-Independent Mechanism of Invasion. mBio 7 (2016).

34. F. Rivera-Chávez et al., Salmonella uses energy taxis to benefit from intestinal inflammation. PLoS Pathog 9, e1003267 (2013).

35. B. Stecher et al., Flagella and chemotaxis are required for efficient induction of Salmonella enterica serovar Typhimurium colitis in streptomycin-pretreated mice. Infect Immun 72, 41384150 (2004).

36. M. A. Matilla, T. Krell, The effect of bacterial chemotaxis on host infection and pathogenicity. FEMS Microbiol Rev 42 (2018).

37. S. P. Nuccio et al., SIMPLE approach for isolating mutants expressing fimbriae. Appl Environ Microbiol 73, 4455-4462 (2007).

38. K. G. Cooper et al., Regulatory protein HilD stimulates Salmonella Typhimurium invasiveness by promoting smooth swimming via the methyl-accepting chemotaxis protein McpC. Nat Commun 12, 348 (2021).

39. C. García-Fontana et al., The involvement of McpB chemoreceptor from Pseudomonas aeruginosa PAO1 in virulence. Sci Rep 9, 13166 (2019).

40. H. P. McLaughlin, D. L. Caly, Y. McCarthy, R. P. Ryan, J. M. Dow, An orphan chemotaxis sensor regulates virulence and antibiotic tolerance in the human pathogen Pseudomonas aeruginosa. PLoS One 7, e42205 (2012).

41. Y. Choi et al., Plasmid-encoded MCP is involved in virulence, motility, and biofilm formation of Cronobacter sakazakii ATCC 29544. Infect Immun 83, 197-204 (2015).

42. A. P. Chaparro, S. K. Ali, K. E. Klose, The ToxT-dependent methyl-accepting chemoreceptors AcfB and Tcpl contribute to Vibrio cholerae intestinal colonization. FEMS microbiology letters 302, 99-105 (2010).

43. M. Guo, Z. Huang, J. Yang, Is there any crosstalk between the chemotaxis and virulence induction signaling in Agrobacterium tumefaciens? Biotechnol Adv 35, 505-511 (2017).

44. R. Kumar Verma, B. Samal, S. Chatterjee, Xanthomonas oryzae pv. oryzae chemotaxis components and chemoreceptor Mcp2 are involved in the sensing of constituents of xylem sap and contribute to the regulation of virulence-associated functions and entry into rice. Mol Plant Pathol 19, 2397-2415 (2018).

45. S. Kojima et al., The Helix Rearrangement in the Periplasmic Domain of the Flagellar Stator B Subunit Activates Peptidoglycan Binding and Ion Influx. Structure 26, 590-598 e595 (2018).

46. K. Postle, R. A. Larsen, TonB-dependent energy transduction between outer and cytoplasmic membranes. Biometals 20, 453-465 (2007).

47. P. Germon, M. C. Ray, A. Vianney, J. C. Lazzaroni, Energy-dependent conformational change in the TolA protein of Escherichia coli involves its N-terminal domain, TolQ, and TolR. J Bacteriol 183, 4110-4114 (2001).

48. A. Jahan-Mihan, B. L. Luhovyy, D. El Khoury, G. H. Anderson, Dietary proteins as determinants of metabolic and physiologic functions of the gastrointestinal tract. Nutrients 3, 574-603 (2011).

49. N. van der Wielen, P. J. Moughan, M. Mensink, Amino Acid Absorption in the Large Intestine of Humans and Porcine Models. J Nutr 147, 1493-1498 (2017).

50. H. G. Windmueller, A. E. Spaeth, Respiratory fuels and nitrogen metabolism in vivo in small intestine of fed rats. Quantitative importance of glutamine, glutamate, and aspartate. J Biol 
bioRxiv preprint doi: https://doi.org/10.1101/2021.05.14.443827; this version posted May 15, 2021. The copyright holder for this preprint

(which was not certified by peer review) is the author/funder. All rights reserved. No reuse allowed without permission.

\section{Chem 255, 107-112 (1980).}

51. Z. L. Dai, G. Wu, W. Y. Zhu, Amino acid metabolism in intestinal bacteria: links between gut ecology and host health. Front Biosci (Landmark Ed) 16, 1768-1786 (2011).

52. S. P. Claus et al., Systemic multicompartmental effects of the gut microbiome on mouse metabolic phenotypes. Mol Syst Biol 4, 219 (2008).

53. B. D. Nguyen et al., Import of Aspartate and Malate by DcuABC Drives $\mathrm{H}_{2} /$ Fumarate Respiration to Promote Initial Salmonella Gut-Lumen Colonization in Mice. Cell Host Microbe 27, 922-936 e926 (2020).

54. R Core Team (2021) R: A language and environment for statistical computing. (R Foundation for Statistical Computing, Vienna, Austria).

55. M. Fischer, S. Zilkenat, R. G. Gerlach, S. Wagner, B. Y. Renard, Pre- and post-processing workflow for affinity purification mass spectrometry data. J Proteome Res 13, 2239-2249 (2014). 
A
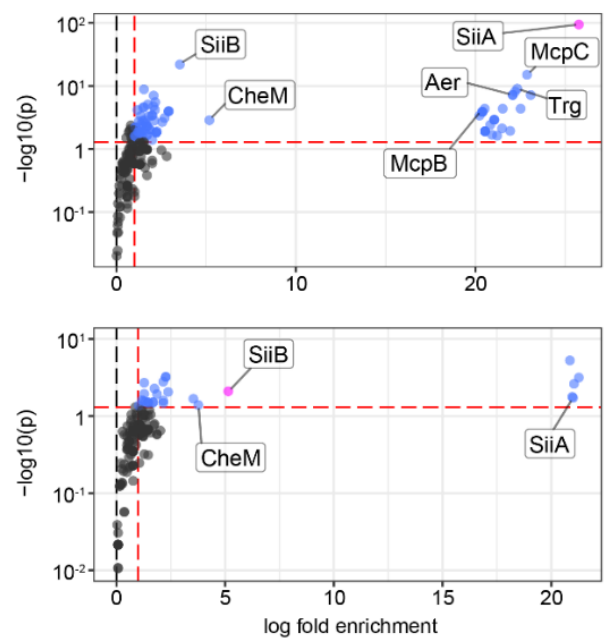

B
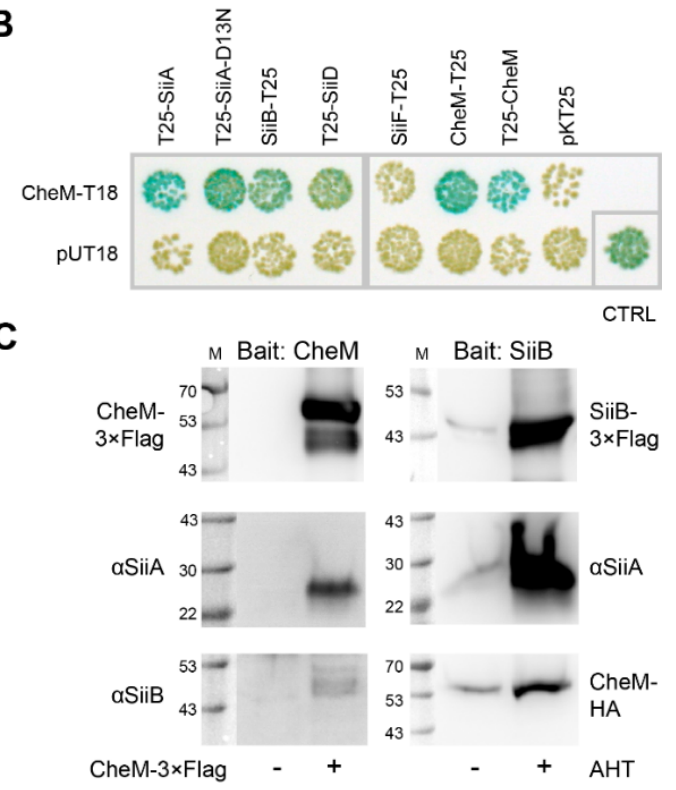

587

588

589

590

591

592

593

594

595

596

597

598

599

600

601

602

603

Figure 1. SPI-4 components interact with CheM. (A) Analysis of affinity purification mass spectrometry data using SiiA-3×Flag (upper panel) or SiiB-3×Flag (lower panel) as bait proteins (magenta dots). Red dashed lines show limits of significant enrichment (>2-fold, $p<0.05)$. Interacting proteins within these limits are depicted in blue with SPI-4 components and MCPs labeled. Summarized data of three independent experiments are shown. (B) Bacterial two hybrid assays evaluating the interaction between the T18 fragment of CyaA alone (pUT18, negative control) or fused to the Cterminus of CheM (CheM-T18) with the CyaA T25 fragment alone (pKT25, negative control) or T25 fused to the indicated SPI-4 proteins or CheM. Functional reconstitution of CyaA activity through proteinprotein interactions resulted in blue color of the E. coli BTH101 reporter strain colonies. A positive control (CTRL) was included based on the interaction of GCN4 leucine zippers. (C) Co-immunoprecipitation using CheM-3×Flag (left panels) or SiiB-3×Flag (right panels) as bait proteins. A plasmid-encoded copy of cheM was expressed from its natural promoter either without (left lane) or with (right lane) $3 \times$ Flag epitope tag. SiiA and SiiB were detected using polyclonal antibodies. Expression of SiiAB-3×Flag from plasmid pWRG905 was induced with addition of $50 \mathrm{ng} / \mathrm{mL}$ anhydrotetracycline (AHT) or left uninduced (left lane). While SiiA was detected with a specific antiserum, a plasmid encoding for CheM-HA was cotransformed allowing CheM detection via $\mathrm{HA}$ tag. $\mathrm{M}=$ molecular weight marker with protein sizes in $\mathrm{kDa}$. 
604

605

606

607

608

609

610

611

612
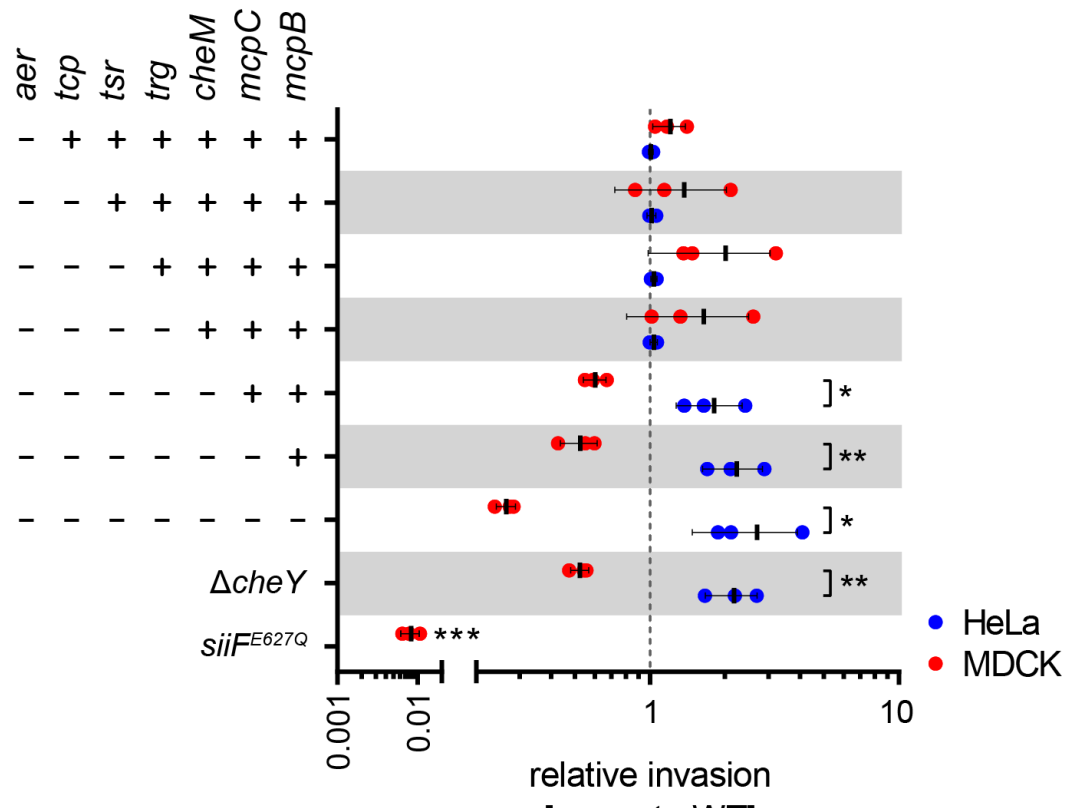

[norm. to WT]

Fig 2. Role of MCPs for bacterial invasion. Relative invasion rates as normalized to $S$. Typhimurium (STM) wild-type (WT, dotted line) after one hour of infection of indicated sequential MCP deletion strains into HeLa (blue) and MDCK (red) cells are shown. The non-chemotactic $\triangle$ cheY strain and a siiF E627Q mutant with a non-functional SPI-4 were included as controls. Data of three independent experiments done in triplicates are depicted. Statistical significance was calculated using unpaired, two-tailed $t$ test between groups or a one sample $t$ test against the hypothetical value 1 (siiF E627Q) and were defined as ${ }^{*}$ for $p<0.05$ and ${ }^{* *}$ for $p<0.01$ and ${ }^{* * *}$ for $p<0.001$. 

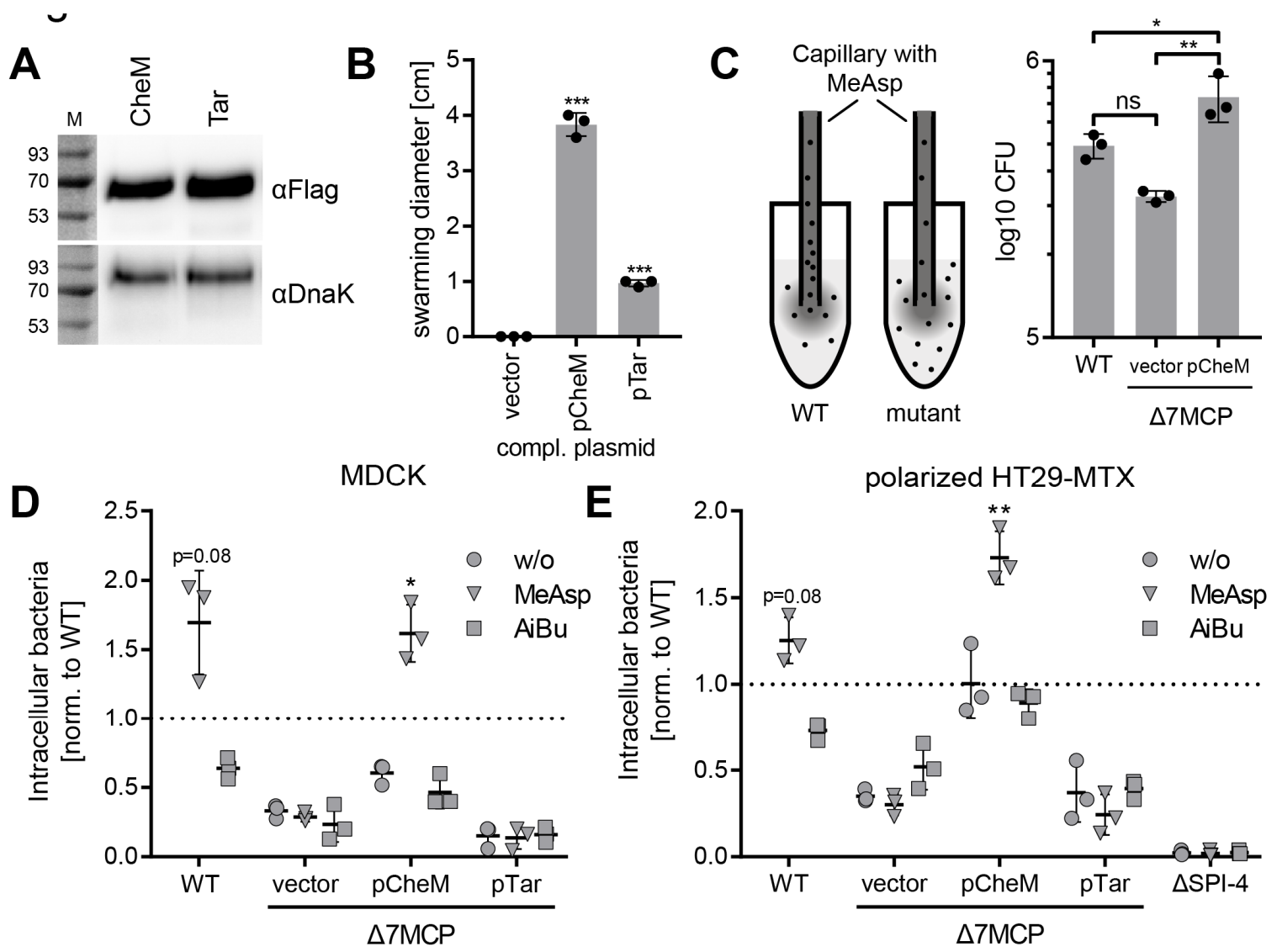

614 Fig 3. CheM complementation and impact of CheM signaling on invasion of polarized cells. (A)

615 Expression of either CheM-3×Flag or Tar-3×Flag from the CheM promoter in a S. Typhimurium (STM) strain lacking all $7 \mathrm{MCP}$ genes $(\triangle 7 \mathrm{MCP})$ was detected with a Flag-specific monoclonal antibody. Equal sample loading was demonstrated by DnaK. $\mathrm{M}=$ molecular weight marker with protein sizes in $\mathrm{kDa}$. (B)

618 The $\triangle 7$ MCP strain transformed with the empty vector pWSK29 (vector) or vectors encoding for CheM 619 (pCheM) or Tar ( $\mathrm{pTar}$ ) were subjected to swarming assays on soft agar plates. Mean swarming diameters $\pm \mathrm{SD}$ after $8.5 \mathrm{~h}$ of growth are depicted for three independent experiments. Statistical significance was calculated using a one sample $t$ test against the hypothetical value 0 (vector control) and were defined as ${ }^{* *}$ for $p<0.001$. (C) Principle of capillary assay. Wild-type (WT) bacteria with functional CheM-dependent chemotaxis swim towards a gradient of $\alpha$-methyl-D, L-aspartate (MeAsp) generated by an attractant-filled capillary. No enrichment within the capillary is observed for mutants with defects in CheM-signaling (left panel). Mean amount \pm SD of STM WT or the $\triangle 7$ MCP with empty vector (vector) or pCheM within the capillary after $1 \mathrm{~h}$. of chemotactic movement from three independent experiments are shown (right panel). One way ANOVA with Tukey's multiple comparison test was calculated and was defined as ${ }^{*}$ for adj. $p<0.05$ and ${ }^{* *}$ for adj. $p<0.01$. (D and E) Relative invasion 
bioRxiv preprint doi: https://doi.org/10.1101/2021.05.14.443827; this version posted May 15, 2021. The copyright holder for this preprint (which was not certified by peer review) is the author/funder. All rights reserved. No reuse allowed without permission.

630 into polarized MDCK (D) or HT29-MTX (E) cells are shown. The strains were either grown without (w/o) 631 attractant or with addition of $10 \mathrm{mM}$ MeAsp or $10 \mathrm{mM} \mathrm{AiBu}$. A SPI-4 deficient strain $(\Delta \mathrm{SPI}-4)$ was 632 included as control for HT29-MTX. Mean \pm SD from three independent experiments are depicted. 633 Statistical significance of strains with increased invasiveness was calculated using a one sample $t$ test 634 against the hypothetical value 1 and were defined as * for $p<0.05$ and ${ }^{* *}$ for $p<0.01$. 


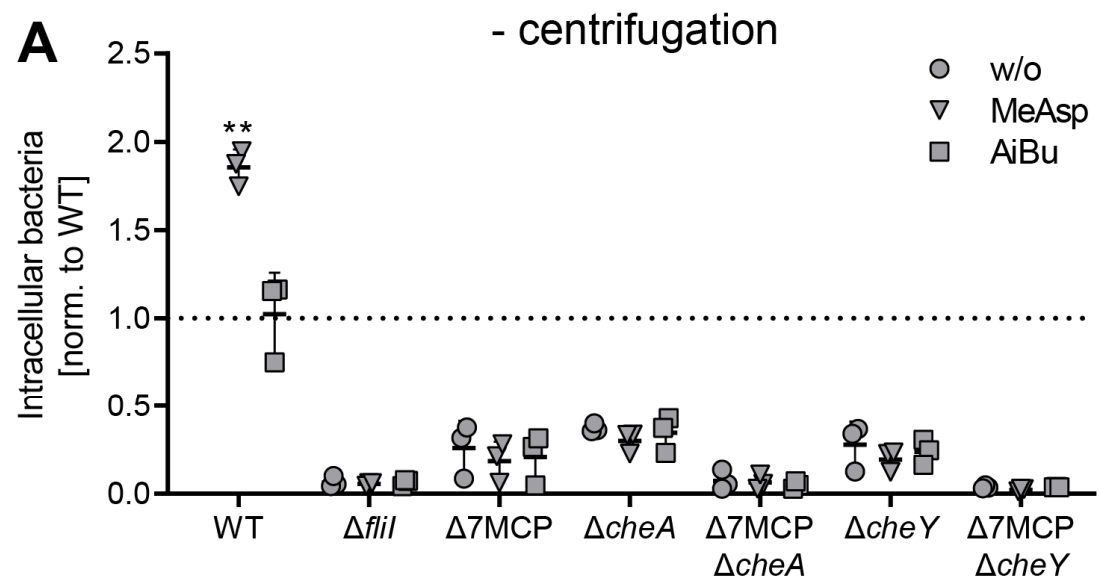

636 Fig 4. Impact of motility and chemotaxis components on Salmonella invasion of polarized MDCK.

637 S. Typhimurium (STM) wild-type (WT), the non-motile $\Delta$ flil mutant, the $\Delta 7$ MCP deletion strain, the 638 chemotaxis mutants $\triangle c h e A$ and $\triangle c h e Y$ or strains lacking besides $c h e A$ or cheY additionally all 7 MCPs 639 were grown without attractant (w/o), in the presence of $10 \mathrm{mM}$ MeAsp or $10 \mathrm{mM} \mathrm{AiBu}$. Inoculi were 640 added to the MDCK cells (A) or bacteria were brought in close host cell contact through centrifugation 641 to compensate for lack of chemotaxis or motility (B). Intracellular bacteria were quantified after one hour 642 of infection and relative invasion rates were calculated based on STM WT without attractant. Data of 643 three independent experiments done in triplicates are depicted. Statistical significance of strains with 644 increased invasiveness was calculated using a one sample $t$ test against the hypothetical value 1.0 and was defined as * for $p<0.05$ and ${ }^{* *}$ for $p<0.01$. 

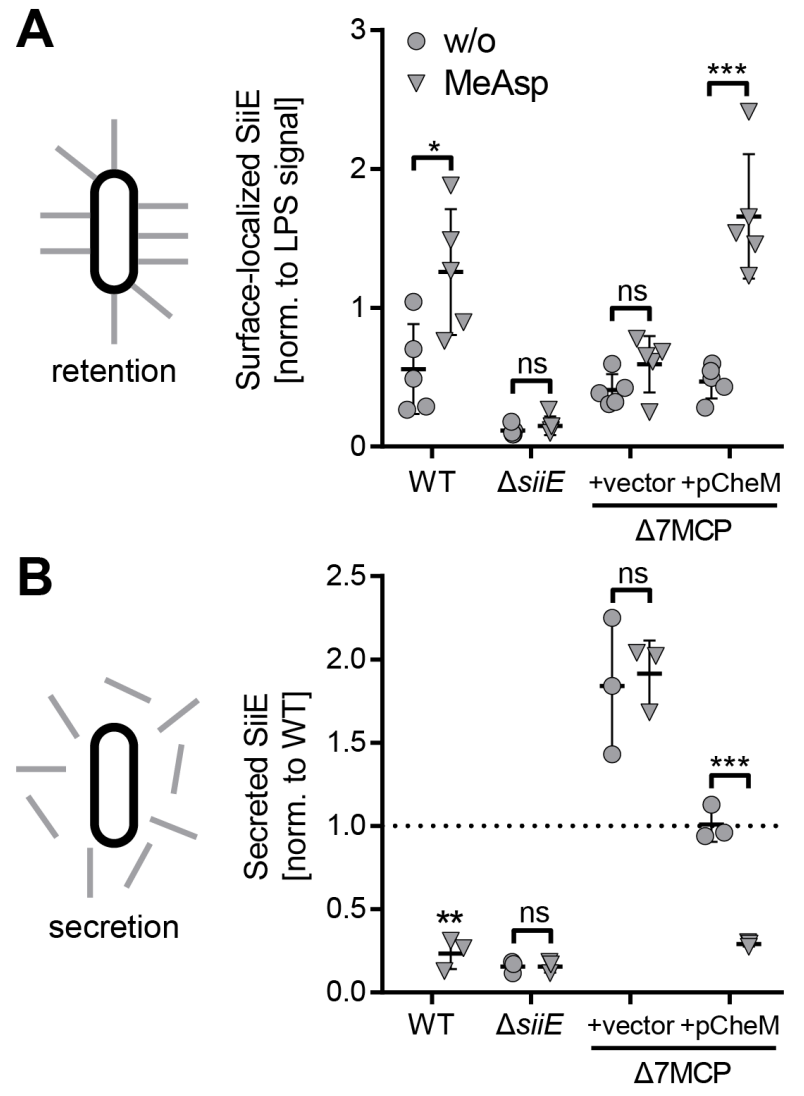

Fig 5. CheM-specific attractant binding promotes SiiE surface localization. (A) Dot-blots were used to quantify surface-localized SiiE and LPS (used for normalization) of S. Typhimurium (STM) wild-type 649 (WT), a $\Delta$ siiE mutant or the $\Delta 7 \mathrm{MCP}$ deletion strain, transformed with the empty vector (vector) or pCheM. Bacteria were either grown without (w/o) or in the presence of $10 \mathrm{mM}$ MeAsp. Mean data \pm SD of five independent experiments are shown. (B) Secreted SiiE was quantified using an ELISA after 3.5 $h$ of growth of the strains and under the conditions as described in (A). Mean data \pm SD of three independent experiments done in triplicates are shown. Statistical significance was calculated using unpaired, two-tailed $t$ test between groups or a one sample $t$ test against the hypothetical value 1.0 (WT in (B)) and was defined as ns $=$ not significant, ${ }^{*}$ for $p<0.05,{ }^{* *}$ for $p<0.01$ and ${ }^{* * *}$ for $p<0.001$. 
A

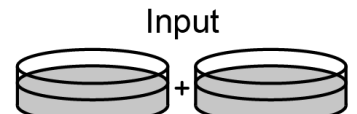

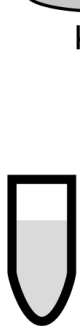

Mix of test and reference strain
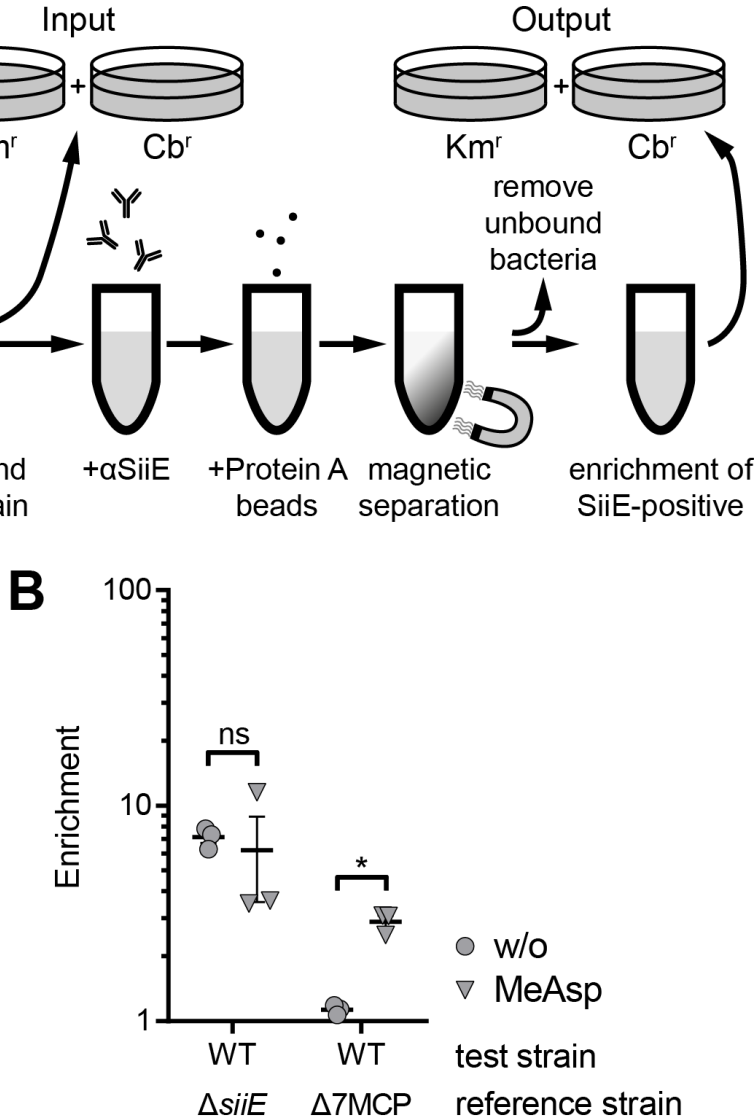

657 Fig 6. Increase of adhesion-competent SiiE in the presence of MeAsp. (A) Principle of a modified screening with immunomagnetic particles for ligand expression (SIMPLE) assay. (B) Enrichment of the test strains compared to the reference strains as indicated without (w/o) or in the presence of $10 \mathrm{mM}$ MeAsp was determined using the SIMPLE assay as shown in (A). Data of three independent experiments done in triplicates are shown. Statistical significance was calculated using unpaired, twotailed $t$ test between groups as indicated and was defined as $\mathrm{ns}=$ not significant and ${ }^{*}$ for $p<0.05$. 

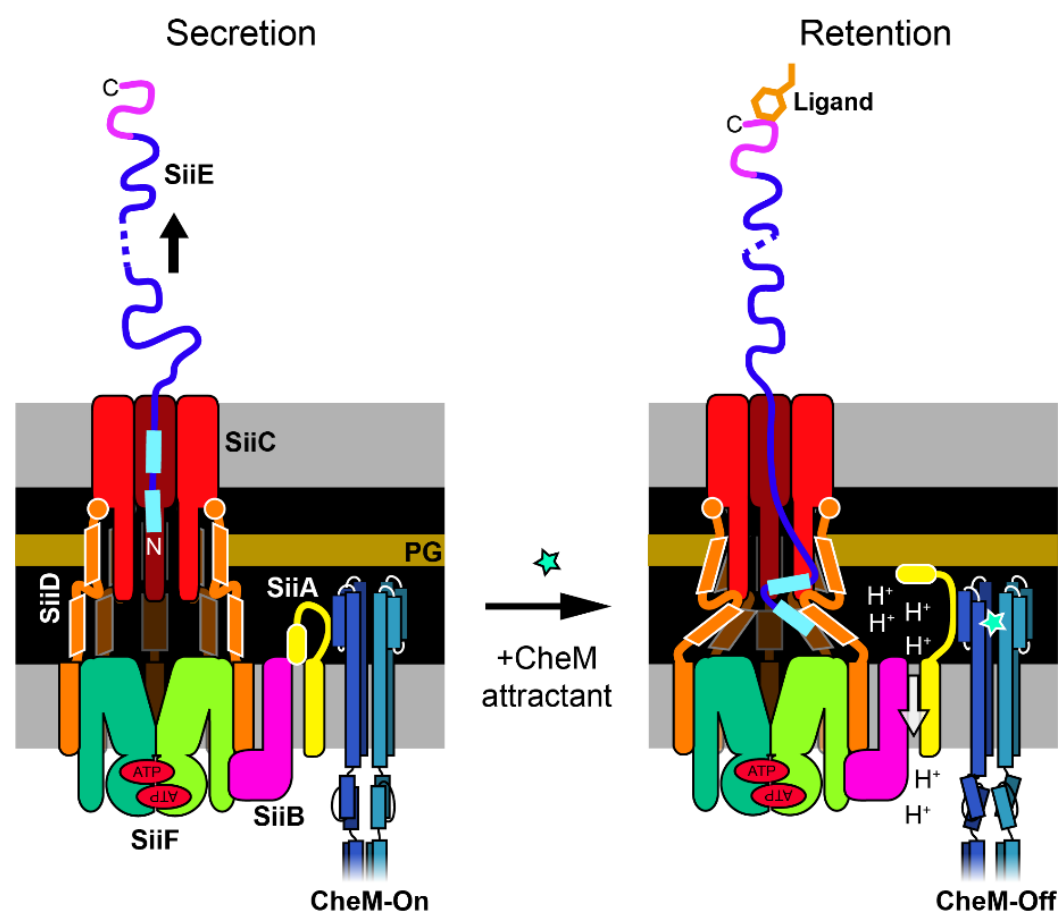

Figure 7. Proposed model how CheM could control SiiE-mediated adhesion. In the absence of attractant, CheM is in the kinase "on" state and the SiiAB proton channel is inactive, presumably due to the periplasmic peptidoglycan (PG) binding domain of SiiA functioning as a plug (left panel). Upon addition of CheM attractant, structural changes in the ligand binding domain of CheM induce displacement of the periplasmic SiiA portion and subsequent association with PG. Proton flux through SiiAB could energize structural changes in the T1SS thus retaining the $\mathrm{N}$-terminal domain of SiiE within the channel (right panel). 